\title{
Analysis of Superregenerative Oscillators in Nonlinear Mode
}

\author{
Silvia Hernández, Student Member, IEEE, Almudena Suárez, Fellow, IEEE
}

\begin{abstract}
Superregenerative oscillators in nonlinear mode are investigated in detail using methodologies based on envelope transient, complemented with additional algorithms. A maximumdetection technique is applied to obtain the input-power threshold for nonlinear operation under different implementations of the quench signal. A mapping procedure enables the prediction of hangover and self-oscillation effects. It is based on the detection of the sequence of local maxima in the envelope amplitude after the application of a single input pulse. Using a contour-intersection method, and depending on the analysis time interval, it is possible to quantify the hangover effects and obtain the oscillation boundary, in terms of any two significant parameters. Then, a compact time-variant behavioral model is derived, valid in the absence of hangover and self-oscillation effects. It consists of a single time-variant Volterra kernel and is applicable provided that the amplitude transitions occur outside the sensitivity interval. The various methodologies are tested in a practical FET-based oscillator at $2.7 \mathrm{GHz}$. The prototype has been manufactured and measured, obtaining good agreement with the analysis results.
\end{abstract}

Index Terms — Logarithmic mode, superregenerative oscillator, stability

\section{INTRODUCTION}

A SUPERREGENERATIVE oscillator (SRO), switched on and off by a quench signal, uses the fast growth of the oscillation amplitude during the start-up transient to obtain high gain amplification [1]-[6]. This is achieved with the advantages of a compact size, low consumption and low cost. SROs have been applied to replace costly amplifier chains in receivers [7][10], and, more recently, to implement active transponders [11][13]. During each quench period, the low-frequency quench signal shifts the dominant pair of complex-conjugate poles of the dc solution (at the oscillation frequency) from the left-hand side (LHS) of the complex plane to the right-hand side (RHS) and then back to the LHS. The SRO is sensitive to the input signal only during a time interval, known as sensitivity interval [2], located about the time value at which the poles cross from the LHS to the RHS. Outside this interval, even if these poles are on the RHS when the input signal is introduced, the oscillation does not have time enough to reach non-negligible amplitude before being switched off by the quench signal.

The SRO can operate in either linear or nonlinear mode [2][3], which strongly depends on the waveform and parameters

This work was supported by the Spanish Ministry of Economy and Competitiveness and the European Regional Development Fund (ERDF/FEDER) under the research project TEC2017-88242-C3-1-R. of the quench signal. In linear (nonlinear) mode, the oscillation is quenched before (after) reaching the nonlinear stage of the oscillation build-up transient. As shown in [2], in linear mode the amplitude of the oscillation pulse is proportional to that of the input signal, whereas in the so-called logarithmic mode, the area under the oscillation envelope is approximately proportional to the logarithm of the input amplitude. The nonlinear mode provides a higher oscillation amplitude and is obtained for a broader choice of quench-signal parameters, so it enables a more robust oscillation [5]-[6], [14]-[15]. However, most previous theoretical studies of the SRO operation have been devoted to SROs in linear mode, which is more easily tractable in an analytical manner.

As stated in [2], in normal operation, the SRO output in a given quench cycle must respond only to the incoming signal during that cycle. However, in the presence of hangover effects, the SRO response will be affected by the remnants of the previous oscillation pulses. When applying a single input pulse, two or more output pulses [1]-[2] will be observed, since the oscillation is able to start again when the system enters the subsequent sensitivity periods. In nonlinear mode, the SRO can also exhibit a self-sustained oscillation [2], [16]. This occurs when the oscillation keeps building up from the remnant of the previous pulses, which greatly reduces the sensitivity to the input signal [17].

In this work, a novel methodology for the analysis and synthesis of SROs in nonlinear mode is presented. In these conditions, the use of an analytical method would necessarily involve a substantial degree of approximation, which might prevent a realistic prediction of the circuit behavior. Instead, the investigation will be based on the use of the envelope-transient method as a fundamental simulation tool [18]-[22], complemented with new techniques, implemented on in-house software. The aim is to obtain a global portrait of the SRO performance versus its most relevant parameters. Some of the techniques rely on a mapping procedure, based on the detection of the maxima of output signal. This way each solution is represented in terms of the sequence of output maxima, instead of using the steady-state envelope waveform of the fundamental harmonic component. The mapping procedure enables a simple representation of the steady-state solution versus any parameter, as well as an efficient detection of the qualitative variations of this solution. This is because it implies a reduction of the solution dimension and complexity, in a manner similar

The authors are with Dpto. Ingeniería de Comunicaciones, Universidad de Cantabria., Av. Los Castros 39005, Santander, Spain (e-mail: silvia.hernandez@unican.es; almudena.suarez@unican.es). 
to the dimension-reduction enabled by the Poincaré map [23], which is an essential tool in the analysis of nonlinear dynamical systems. A difference comes from the fact that the mapping is applied here to an envelope-domain waveform (amplitude of the fundamental component of the SRO output signal), instead of the full time-domain waveform.

Among the theoretical contributions of this work are the investigation of the SRO self-oscillation mechanism, shown to be due to the nonlinear behavior of the envelope of the damping function, as well as the investigation of the relationship between the mode of the output pulse, either nonlinear saturated or nonlinear non-saturated, and the kind of oscillation, either periodic or quasi-periodic (or irregular), respectively. A methodology to obtain the oscillation boundary in terms of two relevant parameters is also presented. All the various kinds of behaviors are general and intrinsic to the oscillation quenching and can be expected in any SRO, regardless of its particular topology or technology, with only quantitative variations. The new systematic techniques provide insightful information into the effect of the most significant SRO parameters on its transient response, stability properties and type of steady-state solution, whose distinction is crucial in nonlinear circuits prone to exhibit self-oscillations.

In addition to the above theoretical investigations, a compact model of the SRO in nonlinear mode, valid in the absence of oscillation effects, will be derived. It takes into account that the SRO operation is time variant, due to the effect of the lowfrequency quench signal. The model is an extension to time variant systems of the single-kernel Volterra model in the envelope domain, presented in [24]-[26]. It can also be considered as an extension of the time-variant model in [27] to nonlinear operation. As will be shown, through a proper timing of the input-amplitude variations, the model is usable under any arbitrarily-modulated input signal, which is well suited for system level simulations.

The paper is organized as follows. Section II briefly describes the envelope-transient analysis of the SRO and presents the quantitative technique to distinguish between linear and nonlinear modes. Section III addresses the mapping and contour-intersection techniques to predict hangover effects and self-oscillatory behavior. Section IV presents the new behavioral model for SROs in nonlinear mode, based on a single-kernel time-variant Volterra model

\section{SRO IN NONLINEAR MODE}

\section{A. Summary of envelope-transient formulation}

For convenience, the general circuit-level envelope domain equations, based on the modified nodal approach (MNA), are briefly recalled [18]-[20]. The circuit variables are expressed in a Fourier series with slowly time-varying harmonic terms, in the following general manner:

$$
\bar{x}(t)=\sum_{k=-N H}^{N H} \bar{X}_{k}(t) e^{j k \omega t}
$$

where $\omega$ is the frequency of the input signal. Note that to achieve amplification the frequency $\omega$ must be close to the circuit self-oscillation frequency. The envelope-transient system is formulated as [18]-[20]:

$$
\begin{aligned}
& \bar{F}(\bar{X}(t))+[j \omega] \bar{Q}(\bar{X}(t))+\frac{d \bar{Q}(\bar{X}(t))}{d t}+ \\
& {[D(t)] * \bar{X}(t)+\bar{G}(t)=0}
\end{aligned}
$$

where $\bar{F}$ is the vector containing the time-varying harmonic components (at $k \omega$ ) of the resistive elements, $\bar{Q}$ is an analogous vector, corresponding to the reactive elements, $[j \omega]$ is the matrix accounting for the derivatives of the complex exponential terms in $(1),[D(t)]$ is the matrix containing the time-varying harmonic components of the impulse responses of the distributed elements, and $\bar{G}$ is the vector of input sources. As explained in [27], this vector contains the low-frequency quench signal $V_{q}(t)$ and the complex envelope $V_{i n}(t)$ of the input signal at the carrier frequency $\omega$, as well as its complex conjugate $V_{i n}^{*}(t)$, at $-\omega$. System (2) is integrated at a much larger time step than the ordinary transient analysis. Its practical resolution through an error-minimization algorithm, after discretizing the time variable, is explained in detail in [18]-[20].

\section{B. Input-power threshold for nonlinear operation}

The SRO behaves in nonlinear mode when the input amplitude is large enough for the oscillator nonlinearity to become relevant. The threshold for nonlinear operation strongly depends on the waveform and parameters of the quench signal $V_{q}(t)$. Here a quantitative analysis methodology is proposed to determine this threshold. For this analysis, a sinusoidal input signal of amplitude $V_{\text {in }}$ at the frequency $\omega$ is considered. On the other hand, the particular waveform of the quench signal (e.g., sinusoidal, sawtooth or square) will be characterized by a set of parameters. For instance, in the case of a sinusoidal waveform, the quench signal is $V_{q}(t)=V_{d c}+V_{p} \cos \left(\omega_{q} t\right)$, so its parameters are $V_{d c}, V_{p}$ and $\omega_{q}$.

The analysis method is based on the detection of the maximum of the amplitude of the SRO output signal, $\max \left|X_{1, \text { out }}(t)\right|$, determined with (2), where the subscript " 1 " indicates the first harmonic component. This maximum will be obtained at a particular time instant of the quench-signal period $T_{q}$. Then, the following ratio is defined:

$$
K\left(V_{\text {in }}\right)=\frac{\max \left|X_{1, \text { out }}\left(V_{\text {in }}, t\right)\right|}{V_{\text {in }}}
$$

In linear mode, this ratio should be constant, in agreement with the derivations in [2], since the output pulse is proportional to $V_{\text {in }}$.

To analyze the influence of a particular parameter $\eta$ on the system linearity, a double sweep is performed in $\eta$ (which may correspond to $V_{d c}$ or $V_{p}$, for instance) and $V_{i n}$, performing an envelope-transient analysis, based on (2), at each pair of values $\left(\eta, V_{i n}\right)$. The envelope simulation is carried out for one period of the quench signal $T_{q}$. At each point $\left(\eta, V_{i n}\right)$ of this double sweep, the output amplitude $\left|X_{1, \text { out }}\left(\eta, V_{\text {in }}, t\right)\right|$ is exported. Next, 
one detects the maximum $\max \left|X_{1, \text { out }}\left(\eta, V_{\text {in }}, t\right)\right|$ and calculates the ratio $K\left(\eta, V_{\text {in }}\right)$, using (3).

The described procedure has been applied to the FET-based oscillator in Fig. 1, built in Rogers $4003 \mathrm{C}$ substrate $\left(\varepsilon_{r}=3.55\right.$, $\mathrm{H}=32$ mils). The oscillation frequency is $f_{o}=2.7 \mathrm{GHz}$ and the quench frequency is $f_{q}=7.5 \mathrm{MHz}$. The drain bias voltage is $V_{D S}=1 \mathrm{~V}$. Note that the purpose of the work is not to obtain a state of the art design, but to investigate the nonlinear behavior of SROs in a global and insightful manner. The parameter used for the analysis of the ratio $K\left(\eta, V_{i n}\right)$ is $\eta=V_{d c}$. Fig. 2 presents the variation of $K\left(V_{i n}\right)$, expressed in $\mathrm{dB}$, versus the input power $P_{i n}$ for different values of $V_{d c}$. One can easily distinguish the linear-mode interval, with a constant $K$, and the nonlinear interval, where $K$ decreases with $P_{i n}$. The effect of the $V_{d c}$ on the threshold between these modes is also evidenced. For higher $V_{d c}$, the threshold decreases. This is due to the fact that the critical pair of complex-conjugate poles stay longer on the RHS, so, under a same $V_{i n}$, the oscillation is able to grow for a longer time. For relatively high $V_{d c}$ the behavior is nonlinear from very small input power. In fact, from $V_{d c}=-1.05 \mathrm{~V}$, the SRO exhibits a self-oscillation, in which the output pulses become independent of the input signal. This will be studied in detail in Section III.

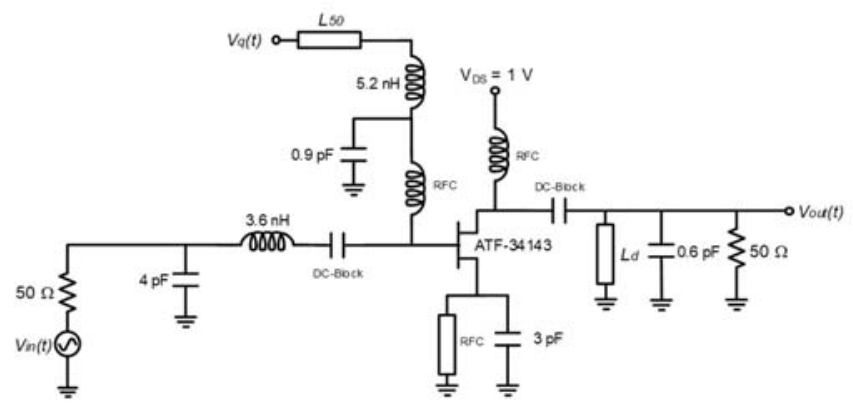

(a)

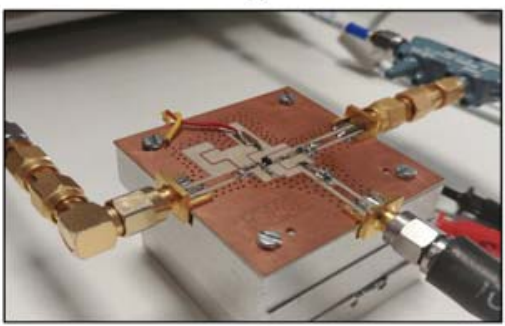

(b)

Fig. 1. FET-based oscillator built in Rogers 4003C substrate $\left(\varepsilon_{r}=3.55\right.$, $\mathrm{H}=32 \mathrm{mils}$ ). The drain bias voltage is $V_{D S}=1 \mathrm{~V}$. The oscillation frequency is $f_{o}=2.7 \mathrm{GHz}$. (a) Schematic. (b) Photograph of the measured prototype.

In the experimental characterization of the SRO, the input signal is generated with the ANRITSU MG3710A Vector Signal Generator setting $f_{\text {in }}=2.7 \mathrm{GHz}$, the sinusoidal quench signal is obtained with the Agilent $81180 \mathrm{~B}$ Arbitrary Waveform Generator and the output signal is measured with a DSO90804A Digital Storage Oscilloscope. Measurement results are superimposed in Fig. 2 for constant $f_{q}=7.5 \mathrm{MHz}$ and $V_{p}=0.3 \mathrm{~V}$, under variations of $V_{d c}$ between $-1.09 \mathrm{~V}$ and
$-1.07 \mathrm{~V}$. It is not possible to consider too small input power due to the presence of noise effects. The experimental measurements are in good qualitative agreement with the analysis predictions. There is a region with flat $K$ and a corner from which $K$ decreases with $P_{i n}$. The corner decreases with $V_{d c}$ as predicted with simulations. Measurements for the lower $P_{\text {in }}$ values are subject to higher error, which explains the fluctuations observed.

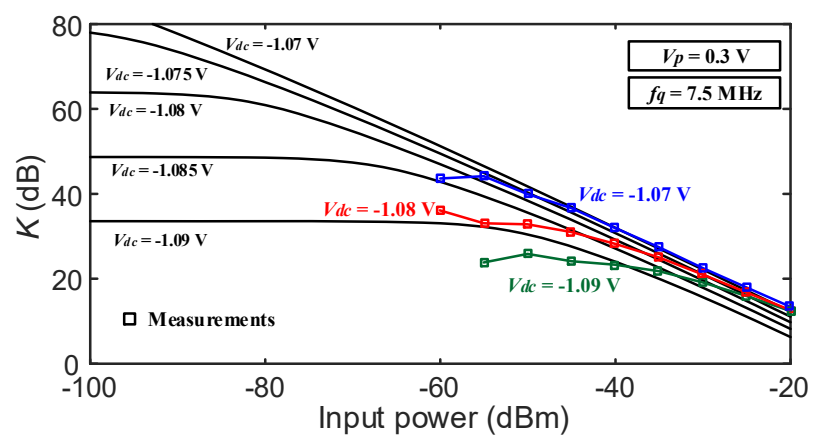

Fig. 2. Prediction of the threshold for nonlinear operation in the FET-based SRO. This is done by evaluating the gain $K$ defined in (3) versus $P_{i n}$. A large $P_{i n}$ range has been considered, with constant $f_{q}=7.5 \mathrm{MHz}$ and $V_{p}=0.3 \mathrm{~V}$, and variations in $V_{d c}$ from $-1.09 \mathrm{~V}$ to $-1.07 \mathrm{~V}$. Measurement results in the same conditions are superimposed.

\section{Influence of the quench frequency}

Another relevant parameter is the quench frequency $\omega_{q}$, due to its strong effect on the time derivatives in (2) and, as a result, on the SRO dynamics. This effect will be analyzed under a given type of quench signal, with a particular waveform and excursion. For instance, in the case of a sinusoidal quench signal, such as $V_{q}(t)=V_{d c}+V_{p} \cos \left(\omega_{q} t\right)$, one will keep $V_{d c}$ and $V_{p}$ at constant values and modify $\omega_{q}$. To quantify the influence of $\omega_{q}$, the maximum of the output pulse, $\max \left|X_{1, \text { out }}(t)\right|$, will be calculated versus $\omega_{q}$. This analysis has been applied to the oscillator in Fig. 1, for $V_{d c}=-1.08 \mathrm{~V}$ and $V_{p}=0.3 \mathrm{~V}$, with the results shown in Fig. 3(a). In the shadowed region, the SRO exhibits a self-oscillation, as will be shown Section III. When decreasing $f_{q}$, the amplitude tends to a saturation value that can be accurately determined as described in the following.

To calculate the saturated output amplitude of the first harmonic of the SRO output pulse, one should replace the quench signal with a dc signal $V_{q}$. This signal will be varied in the voltage in the excursion of the quench signal, easily determined from the quench-signal waveform. For instance, in the case of the sinusoidal waveform $V_{q}(t)=V_{d c}+V_{p} \cos \left(\omega_{q} t\right)$, the excursion is $V_{d c}-V_{p}$ to $V_{d c}+V_{p}$. Since, under variations of the dc signal $V_{q}$, the circuit is not switched, the oscillation should attain a constant output amplitude $\left|X_{1, \text { out }}\right|$ for each $V_{q}$. A sufficiently large time offset must be used in this analysis, to ensure that the oscillation is in steady state for each $V_{q}$. The resulting curve, $\left|X_{1, \text { out }}\right|$ versus $V_{q}$, is presented in Fig. 3(b). Comparing Fig. 3(a) and Fig. 3(b), when the quench frequency 
tends to zero, $\max \left|X_{1, \text { out }}(t)\right|$ tends to the maximum of $\left|X_{1, \text { out }}\right|$ versus $V_{q}$. On the other hand, when $\omega_{q}$ increases, the oscillation has shorter time to build up before being quenched again, so the output pulse is unable to reach the saturation amplitude. This explains the pulse amplitude reduction with $\omega_{q}$, observed in Fig. 3(a).

Fig. 3(c) presents the first output pulse traced versus time normalized to one quench period $T_{q}$, obtained for different values of the quench frequency $f_{q}$. For $f_{q}=2 \mathrm{MHz}$ and $f_{q}=3 \mathrm{MHz}$, the pulse reaches the saturation amplitude. For $f_{q}=6 \mathrm{MHz}$ and $f_{q}=7.5 \mathrm{MHz}$, the pulse reaches the nonlineartransient stage, with non-saturated amplitude. For $f_{q}=9.5 \mathrm{MHz}$, the SRO behaves in linear mode.
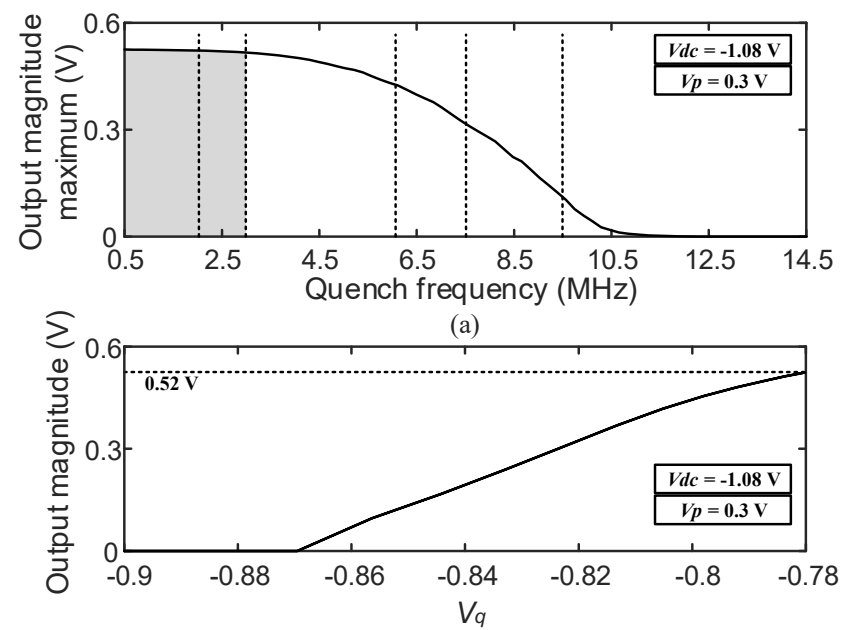

(b)

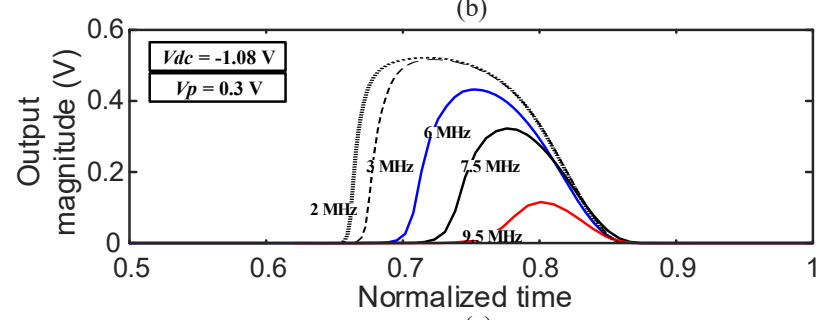

(c)

Fig. 3. Analysis of the effect of the quench frequency $f_{q}$ on the SRO output pulse. (a) Evolution of $\max \left|X_{1, \text { out }}\left(\eta, V_{\text {in }}, t\right)\right|$ versus $f_{q}$. (b) Variation of the maximum of the amplitude of the first harmonic component of the output signal versus the dc voltage that replaces the quench signal, denoted as $V_{q}$. This is varied in the voltage excursion of the quench signal. (c) First output pulse traced versus time normalized to one quench period $T_{q}$, obtained for different values of the quench frequency $f_{q}$.

The response of the SRO to variations in the input power is different in the three cases of linear, non-saturated nonlinear and saturated nonlinear behavior. In linear mode, the increase of $P_{\text {in }}$ gives rise to an increase of the pulse amplitude, as shown in Fig. 4(a), for $f_{q}=9.5 \mathrm{MHz}$. The two waveforms agree by just multiplying one of them by a scale factor. In non-saturated nonlinear mode, there is an increase of both the pulse width and amplitude, as shown in Fig. 4(b), for $f_{q}=7.5 \mathrm{MHz}$. In saturated nonlinear mode, there is an increase of the pulse width, as shown in Fig. 4(c), for $f_{q}=2 \mathrm{MHz}$.
In nonlinear mode, the area under the envelope is proportional to the logarithm of the input amplitude, as analytically derived in [2], [17], in an approximate manner. This has been validated here in a numerical manner, performing, for each $V_{i n}$, an integration of the envelope first output pulse:

$$
S\left(V_{\text {in }}\right)=\int_{0}^{T_{q}}\left|X_{1, \text { out }}\left(V_{\text {in }}, t\right)\right| d t
$$

Fig. 4(d) presents the variation of $S\left(V_{i n}\right)$ versus $P_{\text {in }}$ for $f_{q}=2 \mathrm{MHz}$ and $f_{q}=7.5 \mathrm{MHz}$. In both cases, the variation is approximately linear, in agreement with [2]. At $f_{q}=2 \mathrm{MHz}$, the input amplitude affects the initial exponential growth, but once the waveform reaches the saturation amplitude, this effect vanishes, so the falling sections are overlapped for all the $V_{\text {in }}$ values, as shown in Fig. 4(c). Thus, the difference in the area $S\left(V_{i n}\right)$ is mostly due the initial exponential growth, as stated in [2]. As shown in Fig. 4(d), the area under the pulse envelope is significantly bigger in the case of saturated operation. For a same increment of $P_{i n}$, there is a much smaller ratio between the resulting area increment and the total area.

The distinction between the three types of response: linear, nonlinear non-saturated and nonlinear saturated will be crucial to understand the SRO self-oscillatory behavior, tackled in the next section.

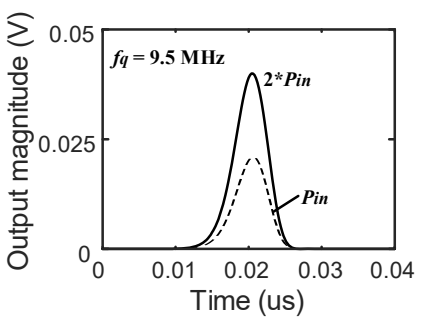

(a)

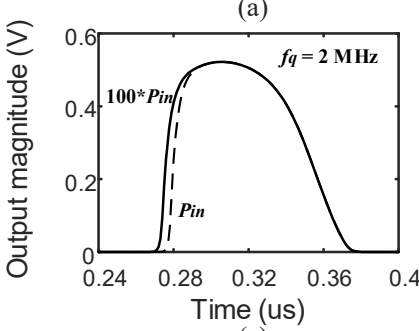

(c)

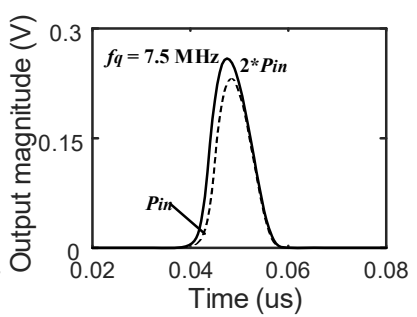

(b)

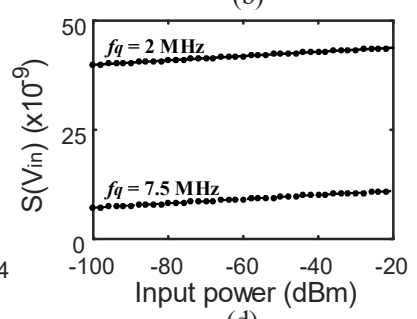

(d)
Fig. 4. Variation of the output pulse with $P_{\text {in }}$ in different regions of the curve $\max \left|X_{1, o u t}\left(\eta, V_{\text {in }}, t\right)\right|$ versus $f_{q}$ [in Fig. 3(a)]. (a) For $f_{q}=9.5 \mathrm{MHz}$. (b) For $f_{q}=7.5 \mathrm{MHz}$. (c) For $f_{q}=2 \mathrm{MHz}$. (d) Variation of $S\left(V_{\text {in }}\right)$ in (4) versus $P_{\text {in }}$ for two values of the quench frequency: $f_{q}=2 \mathrm{MHz}$ and $f_{q}=7.5 \mathrm{MHz}$.

\section{STABILITY ANALYSIS}

\section{A. Self-oscillation mechanism}

In nonlinear mode, the pulses last longer and reach a higher amplitude, so hangover effects [1]-[2] are more likely than in linear mode. In addition, the circuit nonlinear damping may give rise to a self-sustained oscillation in the envelope scale. To illustrate this effect, a cubic nonlinearity oscillator, often used to model the SRO behavior [2]-[3], will be considered (Fig. 5). The nonlinear current is expressed as: 


$$
i(v, t)=a(t) v+b v^{3}
$$

where $a(t)$ is a sinusoidal term, taking positive and negative signs, expressed as $a(t)=A_{d c}+A_{p} \cos \left(\omega_{q} t\right)$, and $b>0$. The second order differential equation that governs the circuit behavior is:

$$
\ddot{v}+\frac{1}{C}\left[G+a(t)+3 b v^{2}(t)\right] \dot{v}+\frac{1}{L C} v=\frac{1}{C} \frac{d i_{i n}(t)}{d t}
$$

where $i_{i n}(t)$ is the input current. The damping term [affecting the time derivative $\dot{v}(t)]$ is:

$$
\zeta(t)=a(t)+G+3 b v^{2}(t)
$$

In the absence of a nonlinear dependence $(b=0)$, the sign of $\zeta(t)$ only depends on $a(t)$ and, thus, on the action of the quench signal. Under nonlinear effects (when $b>0$ ), the damping term $\zeta(t)$ is controlled not only by $a(t)$, but also by the voltage amplitude $|v(t)|$, which gives rise to a feedback mechanism that reduces the negative damping when $|v(t)|$ increases. From the envelope-transient system (2), it is possible to calculate the first harmonic of $v(t)$, given by $V(t)$. Then, the envelope of the damping function can be approximated as: $\zeta_{\text {env }}(t)=a(t)+G+3 b|V(t)|^{2}$, where $|V(t)|$ is the magnitude of the first harmonic. In the following analyses, the sinusoidal input current $i_{i n}(t)$, at the frequency $f_{\text {in }}=2.7 \mathrm{GHz}$, has a limited time duration, within the sensitivity interval. It is implemented by introducing a rectangular pulse $p_{c}(t)$ in the envelope scale, so it is given by $i_{\text {in }}(t)=p_{c}(t) I_{\text {in }} \cos \omega t$.

In a first test, the parameters of the sinusoidal quench signal, at $f_{q}=6 \mathrm{MHz}$, are $A_{d c}=-0.00182 \Omega^{-1}$ and $A_{p}=0.0251 \Omega^{-1}$. Fig. 6(a) presents the amplitude of the first harmonic component of the node-voltage envelope during the first four periods of the quench signal. It exhibits a number of pulses of decaying amplitude, corresponding to hangover effects [2]. Fig. 6(a) also illustrates the double dependence of $\zeta_{\text {env }}(t)$ on $a(t)$ and $|V(t)|$. Note that for $b=0$, and under a sinusoidal quench signal $a(t)$, the envelope of the damping signal $\zeta_{\text {env }}(t)$ is strictly sinusoidal, since it is given by $\zeta_{e n v}(t)=a(t)+G$. In the presence of $b>0$, there will be deviations from this sinusoidal form, caused by the additional dependence on $|V(t)|$, shown in the expression $\zeta_{e n v}(t)=a(t)+G+3 b|V(t)|^{2}$. These deviations are larger for a higher $|V(t)|$. For $A_{p}=0.0251 \Omega^{-1}$ and $b=0$ (linear case), one would obtain a pulse of extremely large amplitude (not represented), since this amplitude keeps growing as long as the envelope of the damping function fulfils $\zeta_{\text {env }}(t)=a(t)+G<0$. However, with $b>0$, the term $\zeta_{\text {env }}(t)$ becomes positive from certain amplitude, which limits the pulse growth. Here the values $b=0.00448 \mathrm{~A} / \mathrm{V}^{2}$ and $G=0.02 \Omega^{-1}$ have been considered. Under the quench signal resulting from $A_{d c}=-0.00182 \Omega^{-1}, A_{p}=0.0251 \Omega^{-1}$, the non-saturated first pulse is only able to excite the system nonlinearity of the second and third pulses. In the fourth pulse, the envelope of the damping function is linear. One obtains a progressive decay to zero of the pulse amplitude, shown in Fig. 6(a). Under the quench signal resulting from $A_{d c}=-0.00182 \Omega^{-1}$, $A_{p}=0.0277 \Omega^{-1}$, the saturated first pulse is able to excite the system nonlinearity in all the subsequent pulses [see Fig. 6(b)], which leads to a self-sustained periodic oscillation. This oscillation has been analyzed for six periods of the quench signal, although only the first four periods are represented in Fig. 6(b) to facilitate the comparison with the other cases.

Under some operation conditions, the pulses in the selfoscillatory solution will not reach the saturation level. Then, the amplitude of each pulse will depend on the remnant value in its corresponding sensitivity interval. If the pulse amplitude is small, the nonlinear effects will be small too, and the initial exponential growth will give rise to a significant variation of the pulse amplitude with the quench period. Thus, the oscillation will be non-periodic. This is illustrated in the analysis of Fig. 6(c), performed for $A_{d c}=-0.00182 \Omega^{-1}$, $A_{p}=0.0261 \Omega^{-1}$ and $f_{q}=6 \mathrm{MHz}$. Small variations in the magnitude of the remnants during the sensitivity period [shown in Fig. 6(c)] give rise to significant variations in the pulse amplitude. The pulses are not identical, so the envelope oscillation can be considered as "unlocked" from the quench signal. Again, the analysis has been carried out for six periods of the quench signal, to assess the periodic or non-periodic nature of the steady-state oscillation, although only the first five periods are represented in Fig. 6(c), to facilitate the comparison. The quite irregular behavior seen in Fig. 6(c) can be expected at the stability boundary. Actually, the oscillation will vanish when slightly increasing $f_{q}$, since this oscillation will not have sufficient time to start up before the quench signal shifts the critical pair of poles to the LHS. On the other hand, when reducing $f_{q}$, the system nonlinearity tends to saturate the pulse amplitudes, which will lead to a periodic oscillation. The $f_{q}$ threshold for the onset of oscillations will depend on the other quench-signal parameters, as well as the particular SRO design. A methodology for the accurate determination of the oscillation threshold in terms of one and two system parameters is given in subsections $C$ and $D$.

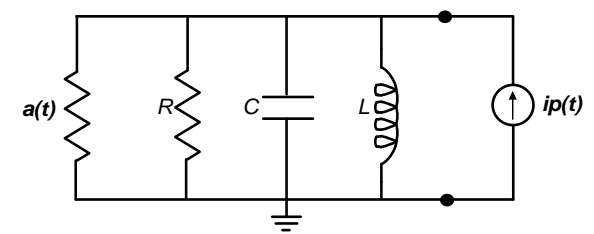

Fig. 5. Cubic-nonlinearity oscillator. Element values are $C=4.03 \mathrm{pF}$, $L=0.86 \mathrm{nH}, R=50 \Omega$ and $a(t)=A_{d c}+A_{p} \cos \left(\omega_{q} t\right)$. The input current $i_{i n}(t)$, at the frequency $f_{\text {in }}=2.7 \mathrm{GHz}$, has a limited time duration, including the sensitivity interval, which is implemented by introducing a rectangular pulse $p_{c}(t)$ in the envelope scale: $i_{i n}(t)=p_{c}(t) I_{i n} \cos \omega t$. The quench frequency is $f_{q}=6 \mathrm{MHz}$ 

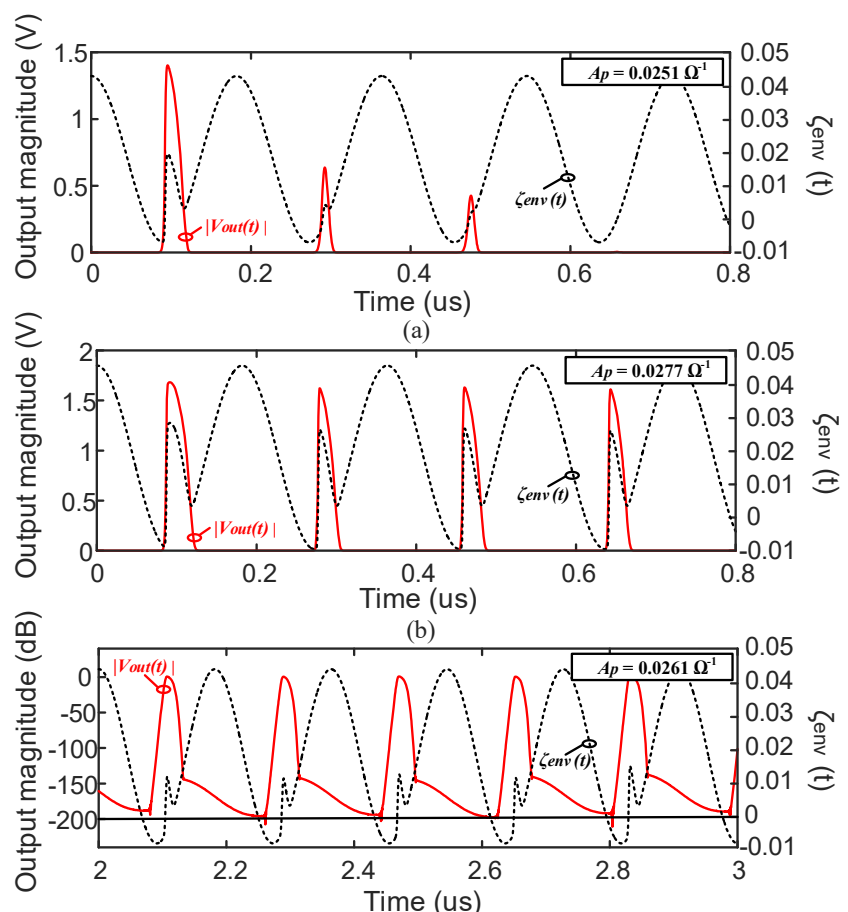

(c)

Fig. 6. Cubic-nonlinearity oscillator at $f_{\text {in }}=2.7 \mathrm{GHz}$ with $f_{q}=6 \mathrm{MHz}$ $A_{d c}=-0.00182 \Omega^{-1}, b=0.00448 \mathrm{~A} / \mathrm{V}^{2}$ and $G=0.02 \Omega^{-1}$. Illustration of the double dependence of $\zeta_{e n v}(t)$ on $a(t)$ and $|V(t)|$ for different $A_{p}$ values. Both $|V(t)|$ (in solid line) and $\zeta_{e n v}(t)$ (in dotted line) are represented. (a) Hangover effects when $A_{p}=0.0251 \Omega^{-1}$. (b) Self-oscillation behavior when $A_{p}=0.0277 \Omega^{-1}$. This oscillation is periodic. (c) Effect of the pulse remnants on the oscillation amplitude for $A_{p}=0.0261 \Omega^{-1}$. This oscillation is quasi-periodic. The output magnitude is represented in $\mathrm{dB}$.

\section{B. Self-oscillation in the FET-based SRO}

When increasing $V_{d c}$ in the gain analysis of Fig. 2, applied to the FET-based SRO, one reaches a value $V_{d c}=-1.05 \mathrm{~V}$ from which there is no longer a flat $K$ interval, which is due to the self-oscillation of the SRO. Instead, $K$ decreases for all the $P_{\text {in }}$ values, as in the case of Fig. 7(a), corresponding to $f_{q}=2 \mathrm{MHz}$, with measurements superimposed. This is because the SRO exhibits a self-sustained oscillation, providing a first output pulse with saturated amplitude, so the gain defined in (3) necessarily decreases with $P_{i n}$. Thus, one can detect unstable behaviors from the inspection of the curve $K$ versus $P_{i n}$.

As in Subsection $A$, for the analysis of the oscillatory behavior, the input signal, at the frequency $\omega$, will only be applied during the first period of the quench signal. In the envelope domain, this will be expressed as: $V_{i n}(t)=p_{c}(t) V_{i n}$. In a first study, the parameter $V_{i n}$ has been swept in the whole interval considered in Fig. 7(a). For $f_{q}=2 \mathrm{MHz}$, the resulting sequence of output pulses is shown in Fig. 7(b). The effect of the input amplitude is only visible in the first oscillation pulses, since this pulse reaches the saturation amplitude. Fig. 7(c) presents a comparison of envelope-domain and transient simulations for $f_{q}=2 \mathrm{MHz}$. Note that the overlap cannot be perfect since the envelope-transient solution is traced in terms of the first harmonic only, though the nonlinear solution contains higher harmonic terms (of smaller amplitude). Fig. 7(d) presents the measurement results.
As shown in subsection $A$, the SRO envelope can also exhibit a non-periodic oscillation, when the pulses are not able to reach the saturation amplitude. This is the case of $f_{q}=5 \mathrm{MHz}$, in Fig. 8. For $f_{q}=7.5 \mathrm{MHz}$, the first output pulse is nonlinear and there are no hangover effects. For $f_{q}=9.5 \mathrm{MHz}$, the behavior is linear.

As has been shown, the SRO can exhibit different forms of behavior when varying a parameter $\eta$, such as the quench frequency $f_{q}$. The next section presents a procedure to efficiently predict the evolution of the SRO behavior versus variations in one or two significant parameters. This will include a prediction of the oscillation boundary.
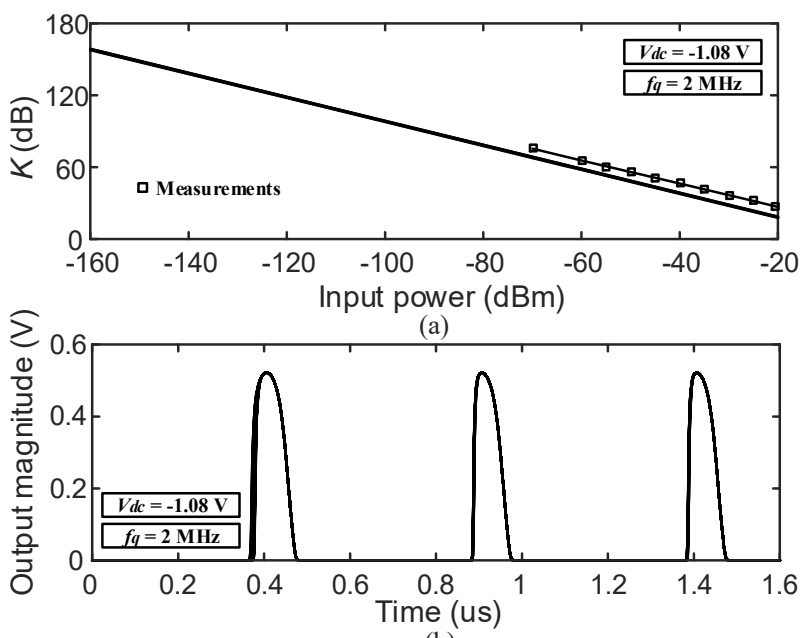

(b)

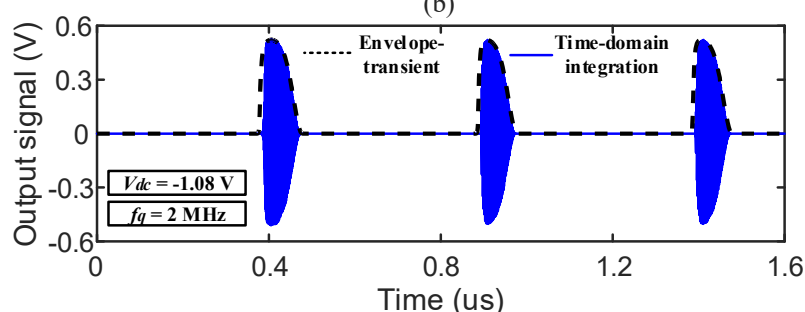

(c)

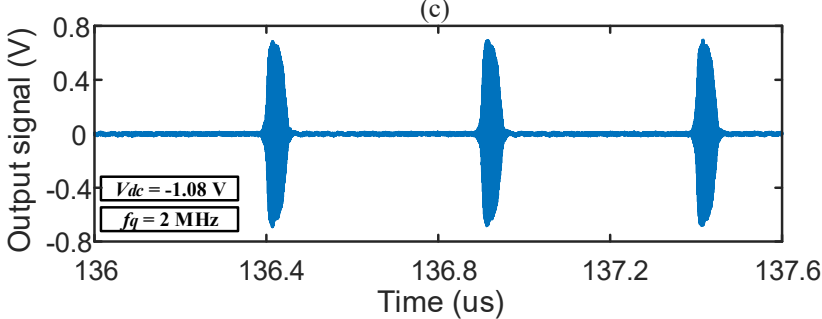

(d)

Fig. 7. Self-oscillation in the FET-based SRO. (a) Variation of the gain $K$ defined in (3) versus $P_{i n}$. (b) Sequences of output pulses obtained for all the $V_{i n}$ values considered in (a). (c) Comparison between envelope-domain and transient simulations for $f_{q}=2 \mathrm{MHz}$. (d) Measured waveform. 


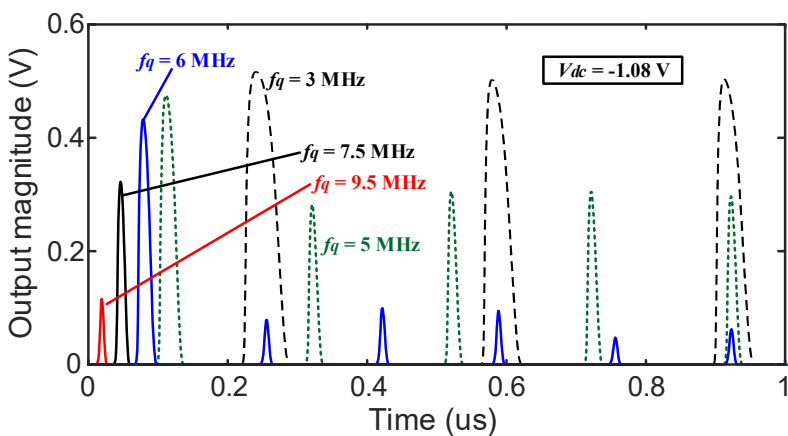

Fig. 8. Output of the FET-based SRO. For $f_{q}=3 \mathrm{MHz}$ there is a self-sustained periodic oscillation. For $f_{q}=5 \mathrm{MHz}$ there is a self-sustained quasi-periodic oscillation. For $f_{q}=6 \mathrm{MHz}$ there is a more irregular oscillation.

\section{Mapping method}

The evolution of the circuit solutions under variations in any parameter $\eta$ can be efficiently analyzed through a mapping method. As in subsections $A$ and $B$, this is based on the application of a single input pulse in the envelope scale, expressed as: $G(t)=p_{c}(t) G_{i n}$. Then, the parameter $\eta$ is varied and the sequence of amplitude maxima of the output signal $X_{1, \text { out }}(t)$, obtained for each $\eta$, is detected. Several periods of the quench signal $M T_{q}$, where $M$ is a positive integer, must be considered. The resulting waveform is exported:

$$
X_{1, \text { out }}\left(t_{n}\right), \quad 0 \leq n \leq N
$$

where the subscript $n$ is a counter of the time instants. The local maxima of the amplitude $\left|X_{1, \text { out }}\left(t_{n}\right)\right|$ are obtained by calculating the time derivative of this amplitude through finite differences:

$$
\dot{X}_{1, \text { out }}^{A}\left(t_{n}\right)=\frac{\left|X_{1, \text { out }}\left(t_{n}\right)\right|-\left|X_{1, \text { out }}\left(t_{n-1}\right)\right|}{t_{n}-t_{n-1}}
$$

which is applied from $n=1$. The superscript $A$ refers to the amplitude of $X_{1, \text { out }}\left(t_{n}\right)$. The set of local maxima must fulfill:

$$
\begin{aligned}
& \dot{X}_{1, \text { out }}^{A}\left(t_{n+1}\right) \dot{X}_{1, \text { out }}^{A}\left(t_{n}\right)<0 \\
& \dot{X}_{1, \text { out }}^{A}\left(t_{n+1}\right)<\dot{X}_{1, \text { out }}^{A}\left(t_{n}\right)
\end{aligned}
$$

For convenience, the set of local maxima will be expressed in terms of an index $i$, used to count the quench periods. Thus, it will be denoted as $X_{1, \text { out }}^{A \text { max }}(i)$, where $i=1$ corresponds to the first maximum. Hangover and self-oscillation effects are analyzed by representing the set of local maxima $X_{1, \text { out }}^{A, \max }(i)$ versus the particular parameter $\eta$.

The method has been applied to the FET-based oscillator in Fig. 1, considering a simulation time interval of $T=8 \mu \mathrm{s}$. When varying the quench frequency, this provides a minimum number $M=7$ of quench signal periods in the whole frequency interval. Fig. 9(a) shows the map of maxima obtained for $V_{d c}=-1.146 \mathrm{~V}$, when varying the quench frequency $f_{q}$. There is only one output pulse for each $f_{q}$ (only one amplitude maximum) so there are no hangover effects. Fig. 9(b) shows the map obtained for $V_{d c}=-1.08 \mathrm{~V}$. For each $f_{q}$ there is a single higher amplitude point, corresponding to the first output pulse, and a set of lower amplitude points, corresponding to the following pulses. The map obtained when considering the time interval $(5 \mu \mathrm{s}, 8 \mu \mathrm{s})$, which avoids the transient effects, has been superimposed on the entire map [obtained when considering the interval $(0 \mu \mathrm{s}, 8 \mu \mathrm{s})]$ using a red color. In the lower frequency range, the SRO envelope oscillates with saturated amplitude, so a single point is obtained. Then, the envelope oscillation unlocks from the quench signal, so there is a transition to a quasi-periodic oscillation, which explains the distribution of points. The width of the pulse distribution increases for amplitudes below $0.15 \mathrm{~V}$, due to a more irregular behavior. The two different operation intervals, with periodic and nonperiodic behavior, have been respectively denoted as $\mathrm{P}$ and NP in Fig. 9(b).

As a final comment, the solution exhibits an invariance with respect to the phase of its self-oscillation components. Thus, under any variation of the initial conditions, there will be a change/translation of the steady-state waveform obtained through the integration of the envelope-transient system. This will give rise to a different distribution of the pulse maxima [Fig. 9(b)]. As a result, there is no continuity of the maxima of a same order (first maximum, second maximum...) when represented versus the parameter.

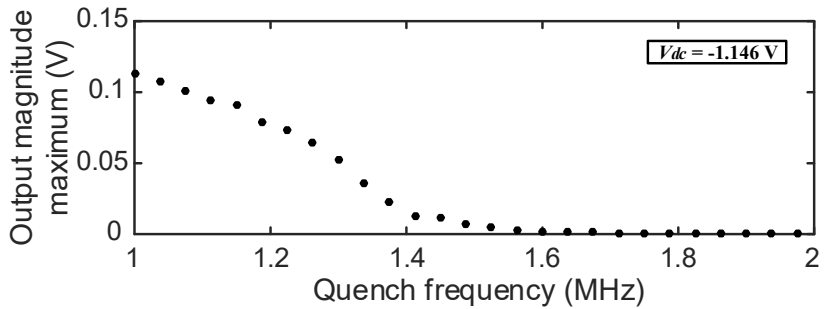

(a)

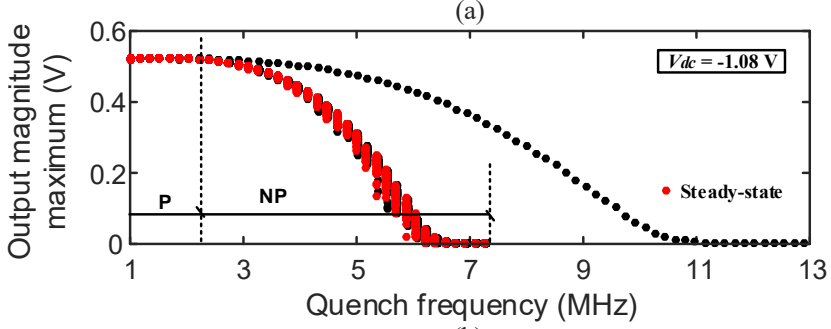

(b)

Fig. 9. FET-based SRO. Mapping method. The simulation time interval considered $(0 \mu \mathrm{s}, 8 \mu \mathrm{s})$. (a) Map of maxima obtained for $V_{d c}=-1.146 \mathrm{~V}$ when varying the quench frequency $f_{q}$. (b) Map of maxima obtained for $V_{d c}=-1.08$ $\mathrm{V}$. For each $f_{q}$, there is a higher amplitude single point, corresponding to the first output pulse, and a set of lower amplitude points, corresponding to the following pulses. The map obtained when considering the time interval $(5 \mu \mathrm{s}$, $8 \mu \mathrm{s})$, which avoids the transient effects, has been superimposed using a red color.

Fig. 10 and Fig. 11 respectively present the simulated and measured results obtained for $V_{D S}=1 \mathrm{~V}, V_{d c}=-1.08 \mathrm{~V}$, $V_{p}=0.3 \mathrm{~V}$ and four values of the quench frequency $f_{q}: 2.1 \mathrm{MHz}$, 3.7 MHz, $6 \mathrm{MHz}$ and $7 \mathrm{MHz}$. The behavior is consistent with the predictions in the map of Fig. 9(b). The experimental measurements in Fig. 11 confirm an amplitude reduction and a tendency to an irregular behavior as $f_{q}$ increases. Note that Fig. 10 presents the amplitude variation of the first harmonic of the output voltage, which is a positive quantity. In contrast, Fig. 11 presents the time-domain variation of the output voltage. There is a good agreement, both qualitatively and quantitatively. In the experimental measurements, the pulses are also affected by 
the noise effects. The irregular behavior obtained in Fig. 10(c) and Fig. 10(d), as well as Fig. 11(c) and Fig. 11(d), is consistent with the observations reported in [28].
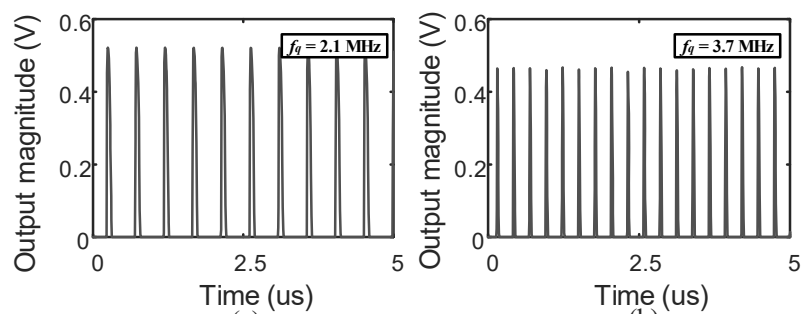

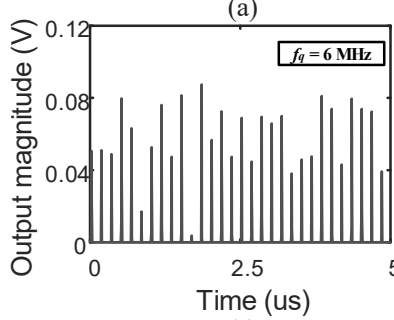

(c)

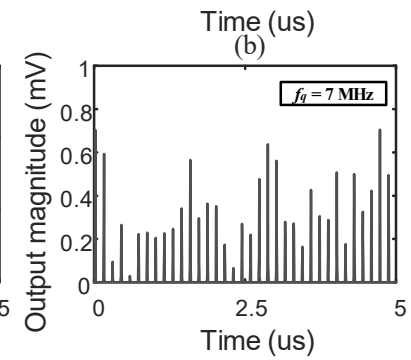

(d)
Fig. 10. FET-based SRO. Variation of the amplitude of the envelope of the output signal for $V_{D S}=1 \mathrm{~V}, V_{d \mathrm{c}}=-1.08 \mathrm{~V}, V_{p}=0.3 \mathrm{~V}$ when considering different values of the quench frequency $f_{q}$. (a) $2.1 \mathrm{MHz}$. (b) $3.7 \mathrm{MHz}$. (c) $6 \mathrm{MHz}$. (d) $7 \mathrm{MHz}$

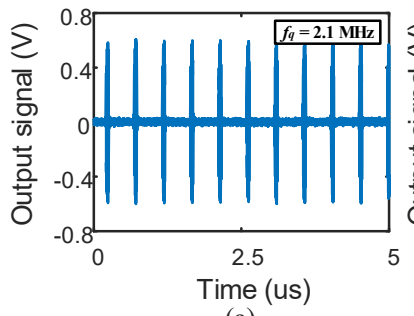

(a)

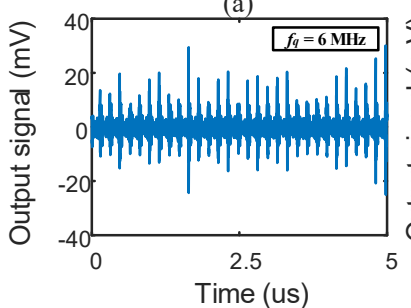

(c)

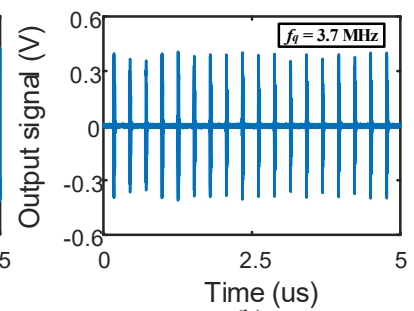

(b)

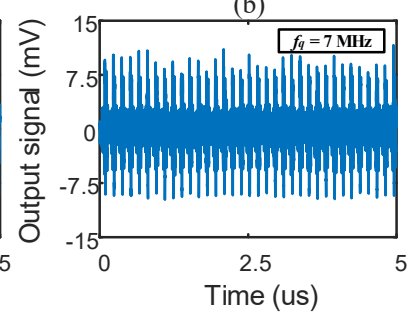

(d)
Fig. 11. FET-based SRO. Measured output-voltage waveforms for $V_{D S}=1 \mathrm{~V}$, $V_{d c}=-1.08 \mathrm{~V}, V_{p}=0.3 \mathrm{~V}$, and different values of the quench frequency $f_{q}$. (a) 2.1 MHz. (b) $3.7 \mathrm{MHz}$. (c) $6 \mathrm{MHz}$. (d) $7 \mathrm{MHz}$.

\section{Envelope-domain contour intersection method}

By means of contour intersections, it will be possible to efficiently predict the effect of two parameters, $\eta_{1}$ and $\eta_{2}$, on the hangover and self-oscillation effects. As in the previous analyses, a single input pulse in the envelope scale is considered, expressed as: $G_{i n}(t)=p_{c}(t) G_{i n}$, which in absolute time corresponds to a sinusoidal signal, at the frequency $\omega$, limited to a fraction of the first quench period. A double sweep is carried out in $\eta_{1}$ and $\eta_{2}$, performing an envelope-transient analysis for each pair $\left(\eta_{1}, \eta_{2}\right)$. The considered integration time interval depends on the analysis goal. This goal can be the detection of the amplitude of second pulse (for $i=2$ ) or the prediction of the oscillation boundary.

$$
\text { a) Contour levels of } X_{1, \text { out }}^{A, \max }(2)
$$

Performing a double sweep in $\eta_{1}$ and $\eta_{2}$ and storing the second maximum $X_{1, \text { out }}^{A, \max }(2)$, in the second quench period interval, $\left(T_{q}, 2 T_{q}\right)$, it is possible to create a 3-D grid, in terms of $\left(\eta_{1}, \eta_{2}, X_{1, \text { out }}^{A \text {,ax }}(2)\right)$. In the plane $\left(\eta_{1}, \eta_{2}\right)$, different contours are obtained from the intersection of this 3-D grid with given amplitude planes $X_{1, \text { out }}^{A \text {, } a x}(2)=P$, where $P$ represents each particular value of the amplitude of the second pulse. Thus, each contour is composed by the set of parameters $\left(\eta_{1}, \eta_{2}\right)$, such that the amplitude of the second pulse is given by $P$.

As an example, Fig. 12 presents the contours $X_{1, \text { out }}^{A, \max }(2)=P$ corresponding to $P=0.1 \mathrm{~V}, P=0.2 \mathrm{~V}, P=0.3 \mathrm{~V}$ and $P=0.4 \mathrm{~V}$ traced in the plane defined by $V_{d c}$ and the quench frequency $f_{q}$. Due to the system continuity, one can expect the hangover effects to disappear when the amplitude of the second pulse tends to zero.

\section{b) Oscillation boundary}

To obtain the self-oscillation boundary, the whole set of steady-state maxima must be considered. This calculation is based on the detection of an incipient steady-state oscillation, satisfying the amplitude condition $X_{1, \text { out }}^{A, \max }=\varepsilon$, where $\varepsilon$ is a sufficiently small number. The boundary will be composed by the set of $\left(\eta_{1}, \eta_{2}\right)$ pairs such that the largest local maximum or maxima of the SRO output signal satisfy $X_{1, \text { out }}^{A, \max }(i)=\varepsilon$, where $i$ is a counter of the maxima fulfilling this amplitude condition. Note that for each pair $\left(\eta_{1}, \eta_{2}\right)$, the whole set of amplitude maxima of the SRO output signal (in steady state) must be analyzed. In the case of a periodic oscillation, the counter $i$ will agree with the number of periods, since all the maxima are identical. However, in the case of a quasi-periodic or chaotic solution, the local maxima of the steady-state output signal will have different values. The condition $X_{1, \text { out }}^{A, \max }(i)=\varepsilon$ will only be fulfilled by the largest maximum (or maxima), so $i$ will have particular integer values, comprised between $i=1$ and the total number of analyzed periods $i=M$.

In this particular application, for the calculation of the oscillation boundary, the time offset is $t_{\text {off }}=5 \mu \mathrm{s}$ and the maximum number of periods of the quench signal is $M_{\max }=22$. The boundary is also represented in Fig. 12, in the plane defined by $V_{d c}$ and the quench frequency $f_{q}$, for $V_{p}=0.3 \mathrm{~V}$. The amplitude threshold used is $\varepsilon=10^{-4} \mathrm{~V}$. Experimental measurements at this boundary are superimposed with very good agreement. The circuit self-oscillates below the boundary, in consistency with the simulated and experimental results of Fig. 10 and Fig. 11. As expected from the system continuity, the amplitude of the second pulse decreases when approaching the boundary. As gathered from Fig. 12, for a less negative dc bias, one should set the quench frequency to higher value to avoid oscillation effects. 


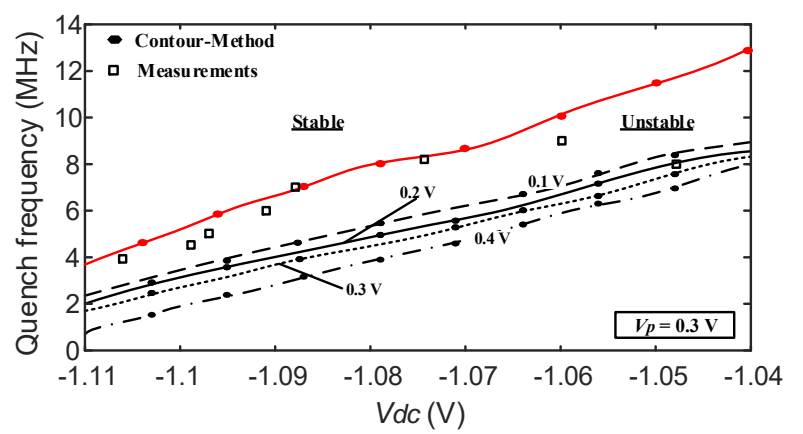

Fig. 12. FET-based SRO with $V_{D S}=1 \mathrm{~V}, V_{d c}=-1.08 \mathrm{~V}, V_{p}=0.3 \mathrm{~V}$. Contours $X_{1, \text { out }}^{A, \max }(2)=P$, corresponding to $P=0.1 \mathrm{~V}, P=0.2 \mathrm{~V}, P=0.3 \mathrm{~V}$ and $P=0.4 \mathrm{~V}$, traced in the plane defined by $V_{d c}$ and the quench frequency $f_{q}$. The steady-state contour $X_{1, \text { out }}^{A, \max }(i)=\varepsilon$, defining the oscillation boundary, has also been represented, with measurement points superimposed.

\section{TIME DOMAIN MODEL OF THE SRO IN NONLINEAR MODE}

When the hangover and oscillation effects have been suppressed, the derivation of a reduced-order model will enable an efficient inclusion of the SRO in system-level simulations. This nonlinear model of the SRO will be an extension to time variant systems of the single-kernel modified Volterra model proposed in [24]-[26]. It can also be considered as an extension of the time-variant model in [27] to nonlinear operation. In this extension, advantage is taken of the fact that the quench signal fully extinguishes the oscillation during a large fraction of each quench-signal period.

\section{A. Summary of the time invariant single-kernel modified Volterra model in [24]-[26]}

As stated in [24]-[26], a time invariant system with negligible long-term dynamics can be modeled with a single kernel modified Volterra model. To extract this single kernel, a sinusoidal envelope signal $V_{i n}(t)=V_{i n} e^{j \Omega t}$ is introduced into the circuit, where $\Omega$ is the envelope frequency. Assuming a band limited response, the output envelope will be $V_{\text {out }}(t)=V_{\text {out }} e^{j \Omega t}$, where $V_{\text {out }}$ varies with the excitation frequency $\Omega$. Performing a double sweep in the envelope amplitude $\left|V_{i n}\right|$ and frequency $\Omega$, the single kernel is calculated as:

$$
H\left(\left|V_{\text {in }}\right|, \omega_{p}+\Omega\right)=\frac{V_{\text {out }}}{V_{\text {in }}}
$$

where $\omega_{p}$ is a suitable carrier frequency, enabling the expression of $H$ as a lowpass function, in terms of the offset frequency $\Omega$. The single kernel in (11) is used to obtain the output envelope $V_{\text {out }}(t)$ resulting from any time-varying input envelope $V_{\text {in }}(t)$, which is calculated as:

$$
V_{\text {out }}(t)=\frac{1}{2 \pi} \int_{-B / 2}^{B / 2} H\left(\left|V_{\text {in }}(t)\right|, \Omega+\omega_{p}\right) V_{\text {in }}(\Omega) e^{j \Omega t} d \Omega
$$

where $B$ is the envelope bandwidth and $V_{\text {in }}(\Omega)$ fulfills:

$$
V_{\text {in }}(t)=\frac{1}{2 \pi} \int_{-B / 2}^{B / 2} V_{i n}(\Omega) e^{j \Omega t} d \Omega
$$

Using convolution, the relationship can be translated to the time domain as [24]-[26]:
$V_{\text {out }}(t)=\frac{1}{2 \pi} \int_{-B / 2}^{B / 2} h\left(\left|V_{\text {in }}(t)\right|, \tau\right) V_{\text {in }}(t-\tau) e^{j \Omega t} d \tau$

where $h$ is the inverse Fourier transform of $H$. In the following, the above single-kernel calculation is extended to the timevariant SRO.

\section{B. Time-variant single-kernel modified Volterra model}

In nonlinear and time variant conditions, the relationship between the envelopes of the output and input signals is the following:

$V_{\text {out }}(t)=\int_{0}^{t} h\left(\left|V_{\text {in }}(t)\right|, t, \tau\right) V_{\text {in }}(\tau) d \tau$

where

$$
h\left(\left|V_{i n}\right|, t, \tau\right)=\frac{1}{2 \pi} \int_{\omega_{\min }-\omega_{p}}^{\omega_{\max }-\omega_{p}} H\left(\left|V_{i n}(t)\right|, t, \Omega+\omega_{p}\right) e^{j \Omega(t-\tau)} d \Omega
$$

The above model will be able to accurately provide the SRO output if the input-amplitude variations take place outside the SRO sensitivity interval, where the system behaves independently of the input signal. This property, together with the full oscillation extinction through a significant fraction of the quench period, will enable the extension of the modified Volterra Kernel to model the time-variant SRO.

The time-variant Kernel $H\left(\left|V_{\text {in }}(t)\right|, t, \Omega+\omega_{p}\right)$ will be calculated for a limited set of discrete amplitude levels, $V_{i n, k}$, where $k=1$ to $K$, comprising those in the multi-level amplitude modulation. At each $V_{i n, k}$, one sweeps the frequency $\Omega$ and obtains the ratio:

$$
H\left(V_{\text {in }, k}, t, \Omega\right)=\frac{V_{\text {out }}(t, \Omega)}{V_{i n, k}}
$$

To obtain the above relationship, the frequency $\Omega$ is swept in the interval $\left(\omega_{\min }-\omega_{p}\right)$ and $\left(\omega_{\max }-\omega_{p}\right)$, and, at each $\Omega$, an envelope-domain integration is performed [27] in the whole time interval considered in the input modulation. Once the set of functions $H\left(V_{i n, k}, t, \omega\right)$ is available, the corresponding collection of impulse responses $h\left(V_{i n, k}, t, \tau\right)$ are obtained in inhouse software. Discretizing the time variable $t$ in (16), as $t_{0}, t_{n}$ $\ldots t_{N}$, the impulse response is calculated as:

$h\left(V_{i n, k}, t_{n}, \tau\right)=\frac{1}{2 \pi} \int_{\omega_{\min }-\omega_{p}}^{\omega_{\max }-\omega_{p}} H\left(V_{i n, k}, t_{n}, \Omega+\omega_{p}\right) e^{j \Omega\left(t_{n}-\tau\right)} d \Omega$

which is done through a suitable combination of $t_{n}$ and $V_{\text {in } k \text {, }}$ following the variations of the input signal. Each time sample $V_{\text {out }}\left(t_{n}\right)$ of the envelope of the output signal is determined through the following convolution integral:

$$
V_{\text {out }}\left(t_{n}\right)=\int_{0}^{t_{n}} h\left(\left|V_{\text {in }}\left(t_{n}\right)\right|, t_{n}, \tau\right) V_{\text {in }}(\tau) d \tau
$$

Then, the envelope $V_{\text {out }}(t)$ is given by the sequence of $V_{\text {out }}\left(t_{n}\right)$ with $t_{0}, t_{n} \ldots t_{N}$. The whole algorithm, implemented in in-house software, is summarized in Table I. Following this method, based on the extraction of the impulse response $h\left(V_{i n, k}, t, \tau\right)$, one can calculate the output to an arbitrarily modulated input signal (with a given set of amplitude levels), which is well suited for system-level simulations. 
The above method has been applied to the circuit in Fig. 1 . Fig. 13 shows the circuit response when varying the input amplitude between three distinct levels. The quench signal parameters are $f_{q}=7.5 \mathrm{MHz}, V_{d c}=-1.08 \mathrm{~V}$ and $V_{p}=0.3 \mathrm{~V}$, which provide a nonlinear response, without hangover and oscillation effects. In a first test [Fig. 13(a)], the amplitude variations never occur during the sensitivity period. The output pulses are overlapped with those obtained with circuit-level envelope-transient simulations. In a second test, some amplitude changes occur during the sensitivity interval [Fig. 13(b)]. In this situation, there are some discrepancies with respect to the circuit-level envelope-transient simulations. They can be avoided through a proper timing of the quench signal.

The SRO behavior is nonlinear since the changes in the input magnitude give rise to a variation in the width of the output amplitude, as shown in Fig. 13(c), where the results of the compact model and circuit-level envelope-domain simulations are overlapped.
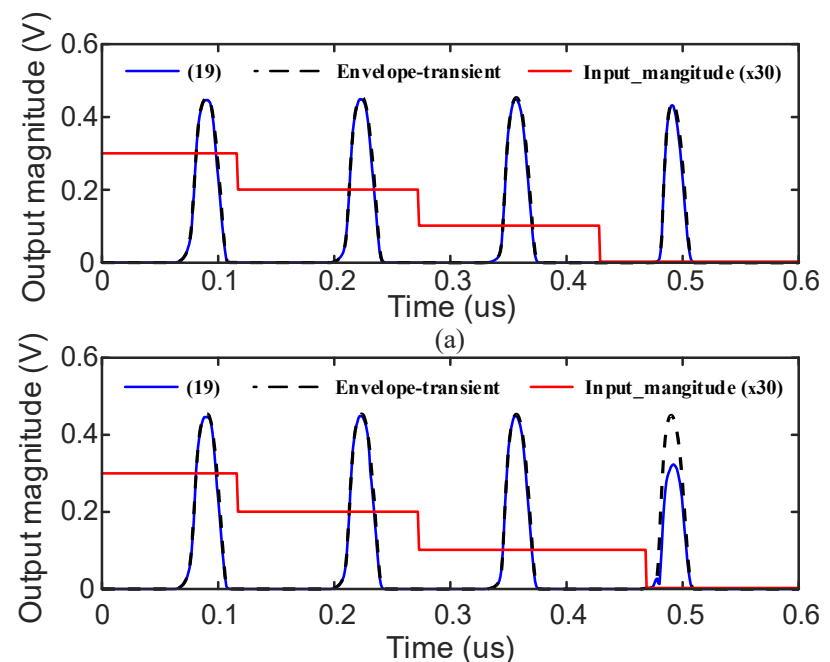

(b)

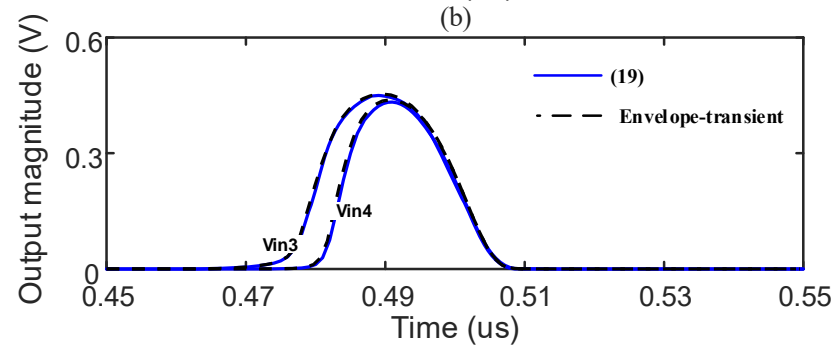

(c)

Fig. 13. FET-based SRO. Response when varying the input amplitude at different quench periods for $f_{q}=7.5 \mathrm{MHz}, V_{d c}=-1.08 \mathrm{~V}$ and $V_{p}=0.3 \mathrm{~V}$. The solution predicted with time-variant behavioral model in (19) is compared with envelope-transient simulations. (a) The amplitude variations never occur during the sensitivity period. (b) Some amplitude changes occur during the sensitivity interval. (c) Expanded view of a single pulse. The new method accurately predicts the increase of the pulse width with the input amplitude.

Fig. 14 shows the output signal when considering an input signal with both phase and amplitude modulations. Fig. 14(a) and (b) compare the variations of the output amplitude and phase obtained with the time-variant single-kernel model and with circuit-level envelope-transient simulations. There is an excellent agreement since the amplitude variations never occur within the sensitivity interval.
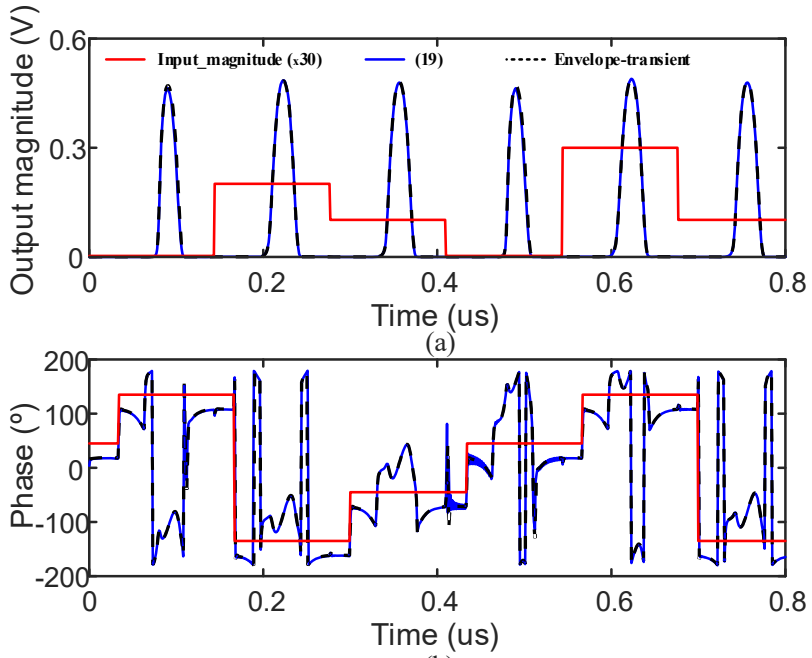

(b)

Fig. 14. FET-based SRO. Response when considering both amplitude and phase modulations. The quench-signal parameters are $f_{q}=7.5 \mathrm{MHz}, V_{d c}=-1.08 \mathrm{~V}$ and $V_{p}=0.3 \mathrm{~V}$. (a) Output amplitude obtained with (19). The solution is compared with circuit-level envelope-transient simulations, with overlapped results. The input amplitude has also been represented. (b) Output phase obtained with (19), compared with the results of circuit-level envelope-transient simulations. The input phase has also been represented

TABLE I

ALGORITHM FOR THE CALCULATION OF THE SRO OUTPUT IN THE LOGARITHMIC MODE

\begin{tabular}{|c|c|}
\hline Read Time_vector & $\begin{array}{l}\text { Reading of the vector of } \\
\text { time values exported from } \\
\text { ADS }\end{array}$ \\
\hline$V_{\text {) }}$ ) & $\begin{array}{l}\text { Construction of the } \\
\text { complex-envelope of the } \\
\text { arbitrarily modulated input } \\
\text { signal }\end{array}$ \\
\hline for $n=1: N$ & $\begin{array}{l}\text { Beginning of the loop that } \\
\text { calculates } V_{\text {out }}\left(t_{n}\right)\end{array}$ \\
\hline $\operatorname{Vin} \_k=\operatorname{Vin}(n)$ & $\begin{array}{l}\text { Detection of the input } \\
\text { amplitude at } t_{n}\end{array}$ \\
\hline $\operatorname{Read} \boldsymbol{H}\left(\boldsymbol{V}_{\mathbf{i n} \_\boldsymbol{H}, \boldsymbol{k}}, \boldsymbol{t}_{\boldsymbol{n}}, \boldsymbol{f}\right)$ & $\begin{array}{l}\text { Reading of the function } \\
f, H\left[V_{i n, k}, t_{n}, 2 \pi\left(f+f_{p}\right)\right]\end{array}$ \\
\hline $\begin{array}{l}H \_V i n \_k= \\
H\left(V_{i n \_H, k}=V i n_{-} k, t_{n}, f\right)\end{array}$ & $\begin{array}{l}\text { exported from ADS. } \\
\text { Construction of the time- } \\
\text { varying transfer function } \\
\text { through a suitable } \\
\text { combination of } t_{n} \text { and } V_{\text {in_k }}\end{array}$ \\
\hline$h \_V i n \_k=I F F\left(H \_V i n \_k\right)$ & $\begin{array}{l}\text { Calculation of the IFFT: } \\
h\left(V_{i n, k}, t_{n}, \tau\right)= \\
\frac{1}{2 \pi} \int_{\omega_{\min }-\omega_{p}}^{\omega_{\max }-\omega_{p}} H\left(V_{i n, k}, t_{n}, \Omega+\omega_{p}\right) e^{j \Omega\left(t_{n}-\tau\right)} d \Omega\end{array}$ \\
\hline
\end{tabular}




\begin{tabular}{|ll||}
\hline $\operatorname{Vout}(n)=$ & Calculation of the \\
$\operatorname{Conv}\left(h \_V i n \_k, V i n \_t\right)$ & convolution integral: \\
& $V_{\text {out }}\left(t_{n}\right)=$ \\
& $\int_{0}^{t_{n}} h\left(V_{\text {in }}\left(t_{n}-\tau\right), t_{n}, \tau\right) V_{\text {in }}\left(t_{n}-\tau\right) d \tau$ \\
end & \\
\hline
\end{tabular}

\section{Measurement results}

The SRO operating in the nonlinear mode has been experimentally tested under an ASK input signal. Fig. 15 shows the photograph of the measurement setup. The modulated input signal is generated using the vector signal generator VSG25A, which provides the power $P_{R F}=-10 \mathrm{dBm}$. The input frequency is $f_{\text {in }}=2.55 \mathrm{GHz}$, which is the maximum one allowed by the VSG25A. Due to the difference with respect to the oscillation frequency $f_{o}=2.7 \mathrm{GHz}$, the required input power levels will be relatively high. At the output of the VSG there is a directional coupler whose coupled output with an attenuation of $10 \mathrm{~dB}$ is connected to the SRO input. Thus, the input power is $P_{i n}=-20 \mathrm{dBm}$. The direct output of the directional coupler allows the measurement of the input signal in a DSO90804A Digital Storage Oscilloscope, which is also used to measure the output signal.

The transistor bias voltage at the drain node is $V_{D S}=1 \mathrm{~V}$. A 2-ASK modulation, with a modulation depth of 0.5 , at the frequency $f_{\text {mod }}=750 \mathrm{kHz}$ has been considered. The quench signal parameters are $V_{d c}=-1.08 \mathrm{~V}$ and $V_{p}=0.7 \mathrm{~V}$, at the frequency $f_{q}=7.5 \mathrm{MHz}$.

Fig. 16 presents the experimental results. Fig. 16(a) shows the input signal after the attenuation of $10 \mathrm{~dB}$. Fig. 16(b) shows a comparison between the results obtained through (19) and those obtained with envelope-transient simulations. Fig. 16(c) compares the experimental results with the predictions by (19) and Fig. 16(d) presents an expanded view of two pulses for two different input amplitudes.

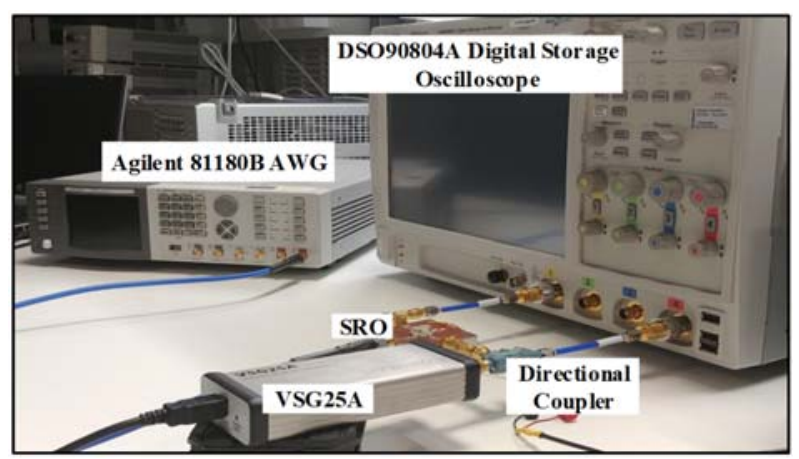

Fig. 15. Photograph of the measurement setup. The RF input signal is generated using the vector signal generator VSG25A, which provides an ASK modulated input signal with the power of $P_{R F}=-10 \mathrm{dBm}$.
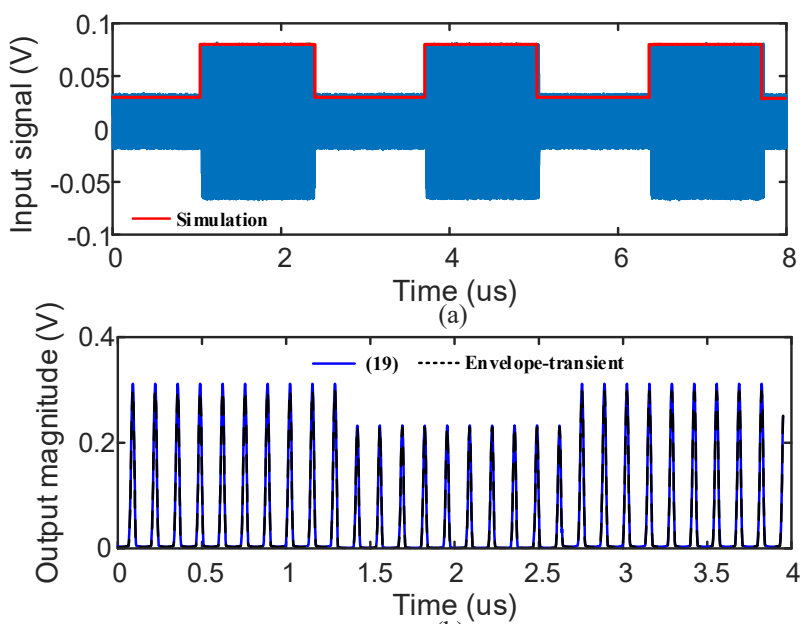

(b)

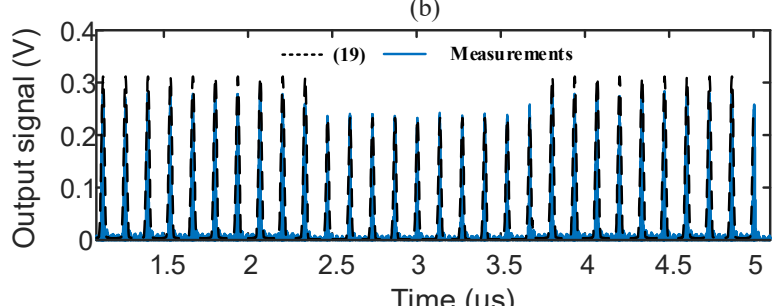

(c)

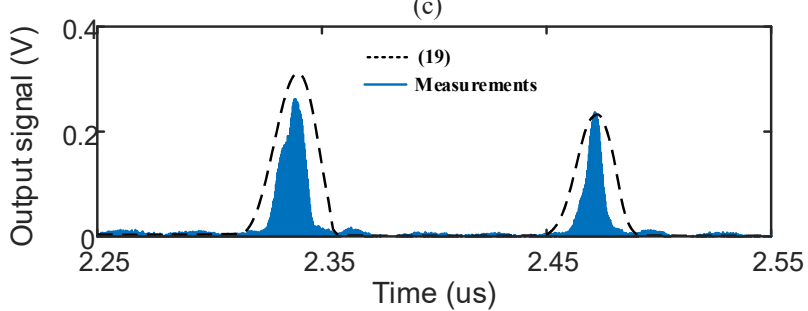

(d)

Fig. 16. Experimental results with an ASK modulated input signal having a modulation depth of 0.5 . (a) Input signal extracted from the transmitted port of the directional coupler, i.e., with an attenuation of $10 \mathrm{~dB}$. (b) Simulation results. Comparison of the output signal obtained by (19) with envelope transient simulations. (c) Comparison of the results provided by (19) with the experimental output. (d) Expanded view of two pulses for different input amplitudes.

\section{CONCLUSION}

An in-depth study of super-regenerative oscillators (SROs) in nonlinear mode has been presented, covering gain, hangover and self-oscillation. A maximum-detection technique enables a quantitative determination of the input-power threshold for nonlinear operation, as well as the distinction between nonlinear saturated, nonlinear non-saturated and linear responses. Then, the self-oscillation mechanism is investigated in detail. This oscillation is shown to arise with a non-periodic behavior, due to the exponential reaction to the asynchronous pulse remnants, and then evolve to a periodic oscillation, when the oscillation envelope is locked by the quench signal. By means of mapping and contour-intersection procedures, oscillation and hangover effects are quantitatively analyzed under the variation of one or two parameters. The procedures enable the calculation of the self-oscillation boundary in terms of two relevant SRO parameters, such as the bias voltage and the quench frequency. A method has also been proposed to obtain a multi-level behavioral model of the SRO, based on the 
calculation of a single time variant Volterra kernel. The model is valid in the absence of self-oscillation and hangover effects, provided that the amplitude changes take place outside the sensitivity interval.

\section{REFERENCES}

[1] J. R. Whitehead, Super-regenerative receivers. Cambridge: University Press, 1950.

[2] F. X. Moncunill-Geniz, P. Pala-Schonwalder and O. Mas-Casals, "A generic approach to the theory of superregenerative reception," in IEEE Trans. Circuits Syst. I, Reg. Papers, vol. 52, no. 1, pp. 54-70, Jan. 2005.

[3] J. Bonet-Dalmau, F. X. Moncunill-Geniz, P. Pala-Schonwalder, F. del Aguila-Lopez and R. Giralt-Mas, "Frequency Domain Analysis of Superregenerative Receivers in the Linear and the Logarithmic Modes," in IEEE Trans. Circuits Syst. I, Reg. Papers, vol. 59, no. 5, pp. 1074-1084, May 2012.

[4] F. X. Moncunill-Geniz, P. Pala-Schonwalder, C. Dehollain, N. Joehl and M. Declercq, "An 11-Mb/s 2.1-mW Synchronous Superregenerative Receiver at $2.4 \mathrm{GHz}$," in IEEE Trans. Microw. Theory Techn., vol. 55, no. 6, pp. 1355-1362, June 2007

[5] P. Pala-Schonwalder, F. X. Moncunill-Geniz, J. Bonet-Dalmau, F. delAguila-Lopez and R. Giralt-Mas, "A BPSK superregenerative receiver. Preliminary results," 2009 IEEE International Symposium on Circuits and Systems, Taipei, 2009, pp. 1537-1540.

[6] P. Palà-Schönwälder, J. Bonet-Dalmau, F. X. Moncunill-Geniz, F. del Águila-López and R. Giralt-Mas, "A Superregenerative QPSK Receiver," in IEEE Trans. Circuits Syst. I, Reg. Papers, vol. 61, no. 1, pp. 258-265, Jan. 2014.

[7] H. Ghaleb, P. V. Testa, S. Schumann, C. Carta and F. Ellinger, "A 160$\mathrm{GHz}$ Switched Injection-Locked Oscillator for Phase and Amplitude Regenerative Sampling," in IEEE Microw. Compon. Lett, vol. 27, no. 9 , pp. 821-823, Sept. 2017.

[8] C. Carlowitz and M. Vossiek, "Demonstration of an efficient high speed communication link based on regenerative sampling," 2017 IEEE MTT-S International Microwave Symposium (IMS), Honololu, HI, 2017, pp. 7174.

[9] P. E. Thoppay, C. Dehollain and M. J. Declercq, "A 7.5mA $500 \mathrm{MHz}$ UWB receiver based on super-regenerative principle," ESSCIRC 2008 34th European Solid-State Circuits Conference, Edinburgh, 2008, pp. 382-385.

[10] V. D. Rezaei, S. J. Shellhammer, M. Elkholy and K. Entesari, "A fully integrated $320 \mathrm{pJ} / \mathrm{b}$ OOK super-regenerative receiver with $-87 \mathrm{dBm}$ sensitivity and self-calibration," 2016 IEEE Radio Frequency Integrated Circuits Symposium (RFIC), San Francisco, CA, 2016, pp. 222-225.

[11] M. Vossiek and P. Gulden, "The Switched Injection-Locked Oscillator: A Novel Versatile Concept for Wireless Transponder and Localization Systems," IEEE Trans. Microw. Theory Techn., vol. 56, no. 4, pp. 859866, Apr., 2008

[12] M. Vossiek, T. Schafer and D. Becker, "Regenerative backscatter transponder using the switched injection-locked oscillator concept," 2008 IEEE MTT-S International Microwave Symposium Digest, Atlanta, GA, 2008, pp. 571-574.

[13] D. R. Frey, "Improved Super-Regenerative Receiver Theory", IEEE Trans. Circuits Syst. I, Reg. Papers, vol. 60, Issue: 12, Pages: $3267-$ 3278, Dec. 2013.

[14] Y. Liu and T. Lin, "A Delta-Sigma Pulse-Width Digitization Technique for Super-Regenerative Receivers," in IEEE Journal of Solid-State Circuits, vol. 45, no. 10, pp. 2066-2079, Oct. 2010.

[15] B. A. Shehhi and M. Sanduleanu, "An 800 $\mu$ W Peak Power Consumption, 24GHz (K-Band), Super-Regenerative Receiver with 200p J/bit Energy Efficiency, for IoT," 2016 29th International Conference on VLSI Design and 2016 15th International Conference on Embedded Systems (VLSID), Kolkata, 2016, pp. 219-223.

[16] R. Hou,"Super-regenerative Receiver for UWB-FM", M.S. Thesis, Department of Microelectronics, Delft University of Technology, The Netherlands, 2008. [Online]. Available: https://repository.tudelft.nl/islandora/object/uuid:73f81186-198d$\underline{4 \text { de9.../download }}$

[17] F. R. W. Strafford, "The Super-Regenerative Detector: an Analytical and Experimental Investigation," in Journal of the Institution of Electrical
Engineers - Part III: Radio and Communication Engineering, vol. 93, no. 21, pp. 23-28, January 1946.

[18] E. Ngoya and R. Larcheveque, "Envelop transient analysis: a new method for the transient and steady state analysis of microwave communication circuits and systems," IEEE MTT-S Int. Microwave Symp. Dig., San Francisco, CA, USA, 1996, vol.3, pp. 1365-1368.

[19] H. Brachtendorf, G. Welsch, R. Laur and A. BunseGerstner, "Numerical Steady-State Analysis of Electronic Circuits Driven By Multi-Tone Signals", Electrical Engineering: 79, pp.103-112, 1996.

[20] E. Ngoya, J. Rousset and D. Argollo, "Rigorous RF and microwave oscillator phase noise calculation by envelope transient technique," 2000 IEEE MTT-S International Microwave Symposium Digest (Cat. No.00CH37017), Boston, MA, USA, 2000, pp. 91-94 vol.1.

[21] K. S. Kundert, "Introduction to RF simulation and its application," in IEEE Journal of Solid-State Circuits, vol. 34, no. 9, pp. 1298-1319, Sep 1999.

[22] J. F. Oliveira and J. C. Pedro, "An Efficient Time-Domain Simulation Method for Multirate RF Nonlinear Circuits," IEEE Trans. Microw. Theory Techn., vol. 55, no. 11, pp. 2384-2392, Nov. 2007.

[23] T. S. Parker and L.O. Chua, Practical Numerical Algorithms for Chaotic Systems, Springer-Verlag, Berlin, 1989.

[24] A. Soury, E. Ngoya and J. M. Nebus, "A new behavioral model taking into account nonlinear memory effects and transient behaviors in wideband SSPAs," 2002 IEEE MTT-S International Microwave Symposium Digest (Cat. No.02CH37278), Seattle, WA, USA, 2002, pp. 853-856 vol.2.

[25] E. Ngoya and A. Soury, "Envelope Domain Methods for Behavioral Modeling", Fundamentals of Nonlinear Behavioral Modeling for RF and Microwave Design. Artech House, 2005.

[26] C. Maziere, A. Soury, E. Ngoya and J. M. Nebus, "A system level model of solid state amplifiers with memory based on a nonlinear feedback loop principle," 2005 European Microwave Conference, Paris, 2005.

[27] S. Hernández and A. Suárez, "Envelope-Domain Analysis and Modeling of Super-Regenerative Oscillators," in IEEE Trans. Microw. Theory Techn., vol. 66, no. 8, pp. 3877-3893, Aug. 2018.

[28] D. M. W. Leenaerts, "Chaotic behavior in super regenerative detectors," in IEEE Trans. Circuits Syst. I, Fundam. Theory Appl, vol. 43, no. 3, pp. 169-176, March 1996.

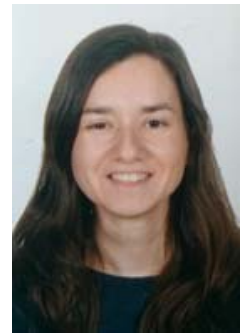

Silvia Hernández (S'17) was born in the Canary Islands, Spain. She received her M.S. degree in Telecommunication Engineering from the University of Las Palmas de Gran Canaria, Canary Islands, Spain, in 2015. The same year, she entered the Institute for the Technological Development and Innovation in Communications, University of Las Palmas de Gran Canaria, as a research assistant. In 2016, she joined the Communications Engineering Department, University of Cantabria, where she is currently working towards her Ph.D. degree.

Her research interests include stability, phase-noise analysis and the study of new methods for the analysis and design of nonlinear microwave circuits.

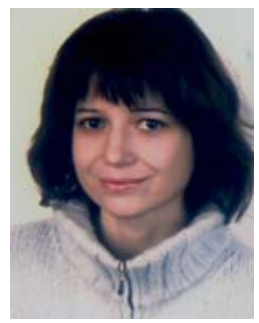

Almudena Suárez (M'96-SM'01-F'12) was born in Santander, Spain. She received the degree in electronic physics and the Ph.D. degree from the University of Cantabria, Santander, Spain, in 1987 and 1992, respectively, and the Ph.D. degree in electronics from the University of Limoges, France, in 1993. At present, she is a Full Professor at the University of Cantabria, and a member of its Communications Engineering Department. She has authored the book Analysis and design of autonomous microwave circuits (IEEE-Wiley,2009) and coauthored the book Stability analysis of microwave circuits (ArtechHouse,2003). She belongs to the technical committees of the IEEE International Microwave Symposium and European Microwave Conference. She was an IEEE Distinguished Microwave Lecturer for the period 2006-2008. She is an associate editor of the IEEE Microwave Magazine. She is a member of the Board of Directors of EuMA and the Editor in Chief of International Journal of Microwave and Wireless Technologies from Cambridge University Press. 